\title{
Herramienta para el diseño de sistemas de posicionamiento tridimensional usados en fabricación digital
}

\section{Software tool for design of three-dimensional positioning systems used in digital manufacturing}

\author{
Jersson Xavier León-Medina' \\ Edgar Absalón Torres-Barahona ${ }^{2}$
}

Recibido: junio 09 de 2015

Aceptado: septiembre 22 de 2015

\begin{abstract}
Resumen
La fabricación digital crea piezas terminadas a partir de modelos diseñados en computador con base en procesos de manufactura CAD-CAM, que abren camino a la flexibilidad y a la productividad en la industria. Este trabajo presenta una herramienta informática que asiste el diseño de equipos de fabricación digital para piezas de geometría compleja a través de control numérico computarizado. Se parte de la identificación de las características de procesos de manufactura susceptibles de automatización y se establece un referente para definir el diseño mecánico, electrónico y de control de un equipo de posicionamiento tridimensional; para ello, a través de una herramienta informática, se definen los parámetros de construcción de los sistemas de posicionamiento, se señalan las variables, las constantes y los criterios, para que mediante la aplicación de teorías referentes al diseño electromecánico, se realicen los cálculos necesarios de los componentes pertenecientes al equipo. Finalmente, una interfaz de usuario permite incorporar los datos, proporcionar los resultados del diseño del equipo y obtener el diseño CAD estructural del sistema de posicionamiento.
\end{abstract}

Palabras clave: fabricación digital, CAD/CAM, herramienta informática, robot cartesiano.

\begin{abstract}
Digital manufacturing creates finished parts from designed models in computer-based manufacturing processes such as CAD-CAM processes, opening the road to flexibility and productivity in industry. This paper presents a software tool that assists the design of digital production equipment, creating complex geometry parts through computerized numerical control. It was started by identifying the characteristics of manufacturing processes capable of automation and it was established a benchmark to define the mechanical, electronic, and control design for three-dimensional positioning equipment. Through a computer tool, the parameters of construction positioning systems were defined, besides, the variables, constants, and criteria were noted, so that by applying theories concerning the electromechanical design, necessary calculations for find the right components belonging to the machine are made. Finally, a user interface allows incorporating data, providing results of the machine design, and getting the structural CAD design of the positioning system.
\end{abstract}

Keywords: digital manufacturing, CAD / CAM, computer tool, Cartesian robot.

\footnotetext{
1 Ingeniero Electromecánico, Magíster en Ingeniería Mecánica, Universidad Pedagógica y Tecnológica de Colombia, Sede Duitama, Colombia. E-mail: jersson.leon@uptc.edu.co

2 Ingeniero Electromecánico, Magíster en Ingeniería Mecánica, Universidad Pedagógica y Tecnológica de Colombia, Sede Duitama, Colombia.E-mail: edgar.torres@uptc.edu.co
} 


\section{Introducción}

A través del uso de sistemas cartesianos de posicionamiento tridimensional, la fabricación digital transforma piezas diseñadas por computador en artículos terminados reales (Chryssolouris, et al., 2009). Dentro de los procesos de manufactura vinculados a la fabricación digital se encuentran: el corte por láser, el corte por plasma, la impresión 3D, el maquinado y la soldadura, que poseen sistemas mecánicos, electrónicos y de control semejantes en su diseño para la ubicación de la herramienta principal en su funcionamiento (Westkämper, 2007).

El presente trabajo permite a los interesados en esta tecnología, apropiar técnicas de producción de equipos de fabricación digital y solucionar problemas reales de la industria; por ejemplo, en el sector metalmecánico de construcción de tanques para transporte de hidrocarburos, los procesos de corte de lámina por oxicorte y plasma son los más susceptibles de automatización (León-Medina, 2014), por lo que la implementación de tecnología de fabricación digital con sistemas cartesianos de posicionamiento tridimensional mejoraría notablemente la velocidad y calidad del proceso.

Debido a que los procedimientos de corte de lámina por oxicorte y plasma corresponden a procesos tridimensionales de movimiento, en donde la herramienta de corte se posiciona en los ejes horizontales $\mathrm{X}$ e $\mathrm{Y}$ dentro de un sistema cartesiano y el movimiento en el eje vertical $Z$ permite controlar la altura de trabajo (Overby, 2010), se procede a restringir los equipos diseñados a una configuración específica de movimiento cartesiano, con movimiento lineal en sus tres ejes.

La herramienta informática desarrollada proporciona la información relacionada con los componentes de cada equipo a diseñar, separados en tres grupos: un sistema mecánico con configuración estructural cartesiana que soporta la herramienta de trabajo quien ejecuta el proceso, un sistema electrónico de control que interpreta señales y envía órdenes a la planta y un sistema informático que transfiere la información desde un computador para la ejecución del proceso, los cuales se seleccionan a partir de criterios de funcionalidad, accesibilidad y costo.

El presente trabajo ilustra la creación de la herramienta informática, contextualizando en primer lugar la fabricación digital y a continuación definiendo los diferentes software que fueron utilizados para su correcto funcionamiento, posteriormente se muestran las partes que componen un equipo cartesiano de posicionamiento en lo concerniente a los sistemas mecánicos y de control para finalmente obtener las especificaciones de un ejemplo de aplicación en un equipo de corte por plasma.

\section{Materiales y métodos}

\subsection{Fabricación digital}

La fabricación digital se basa en un sistema integrado, basado en computador, compuesto de simulación, visualización tridimensional, análisis y diversas herramientas de colaboración para crear definiciones de producto y procesos de fabricación simultáneamente. La fabricación digital ha evolucionado a partir de iniciativas como la fabricación flexible, el diseño para la fabricación (DFM), la fabricación integrada por computador (CIM), el diseño de procesos con tecnología CAD / CAM; que reunidas intervienen en la forma en que distintos productos son desarrollados y fabricados (Wilfert \& Seeland, 1986). Dentro de los procesos de manufactura pertenecientes a la fabricación digital se encuentran los de corte de lámina de metal, los cuales deben responder a requerimientos específicos como: precisión dimensional de los componentes, volumen de producción y cumplir la norma ISO 9013 para cortes térmicos (International Organization for Standardization, 2002).

En la figura 1 se observa un diagrama generalizado de un equipo de corte, donde la planta, (mesa de coordenadas), el sistema de control (software 
y equipo de cómputo), las unidades de potencia (circuitos de control de energía) y los componentes de suministro energético según el principio de actuación (p.ej. oxígeno y propano) están dispuestas para el funcionamiento como un equipo de Control Numérico Computarizado - CNC.

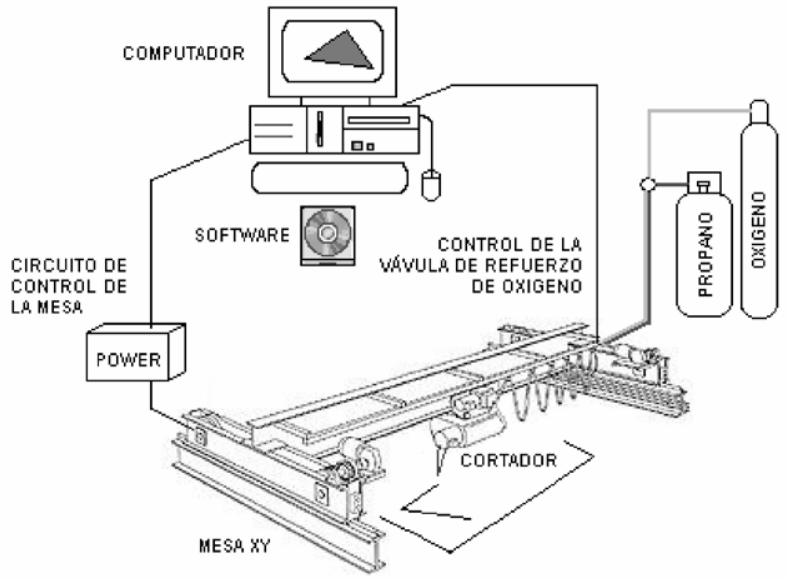

Figura 1. Sistema general del proceso de corte automatizado de lámina (Sarmiento \& Rojas, 2006 p. 45).

2.2 Herramienta de diseño de equipos

La herramienta desarrollada para el diseño de equipos de posicionamiento, funciona a través del uso de un applet Java creado en NetBeans IDE 7.3 que con la ayuda de la librería POI 3.9, (Apache Software Foundation, 2013), se comunica con MS Excel y modifica las dimensiones correspondientes al diseño paramétrico estructural de un sistema de posicionamiento en Solid Edge ST6; además, desde una base de datos selecciona los componentes adecuados del equipo de acuerdo a los datos primarios de entrada introducidos como tamaño, precisión, resolución, mecanismos, motores entre otros requerimientos. En la figura 2 se ilustra la integración de los diversos software utilizados en el proceso de diseño.

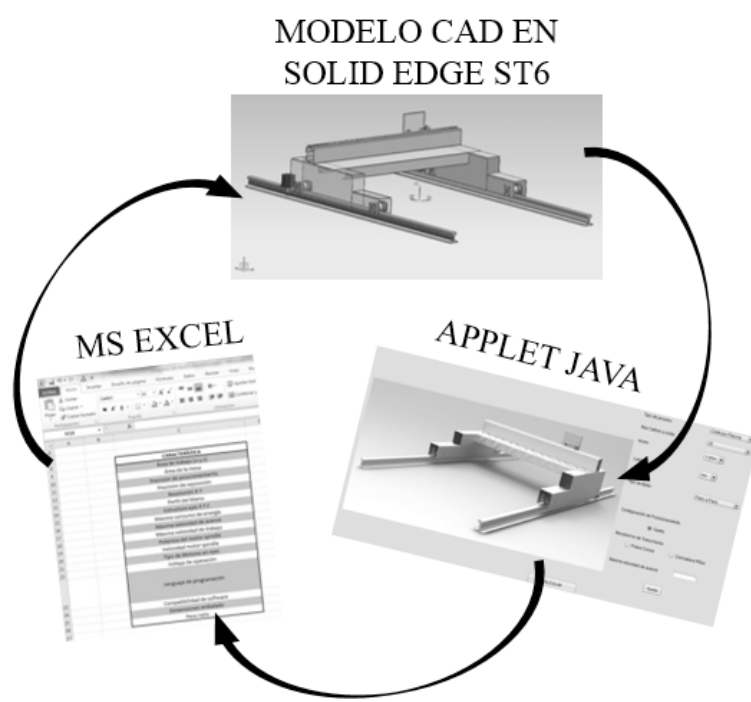

Figura 2. Integración de herramientas en el proceso de diseño de un sistema cartesiano.

\section{Diseño de interfaz gráfica de usuario}

El diseño de la interfaz de usuario permite incorporar los datos y analizar los resultados de los cálculos obtenidos para los sistemas mecánicos, de actuación y de control. Se realiza a partir de la selección del software NetBeans 7.3 y la librería POI 3.9 para establecer la correcta comunicación con el software MS Excel, este último se comunica con Solid Edge, para definir el diseño CAD de la estructura. La interfaz de usuario diseñada en NetBeans 7.3 , se muestra en la figura 3. 


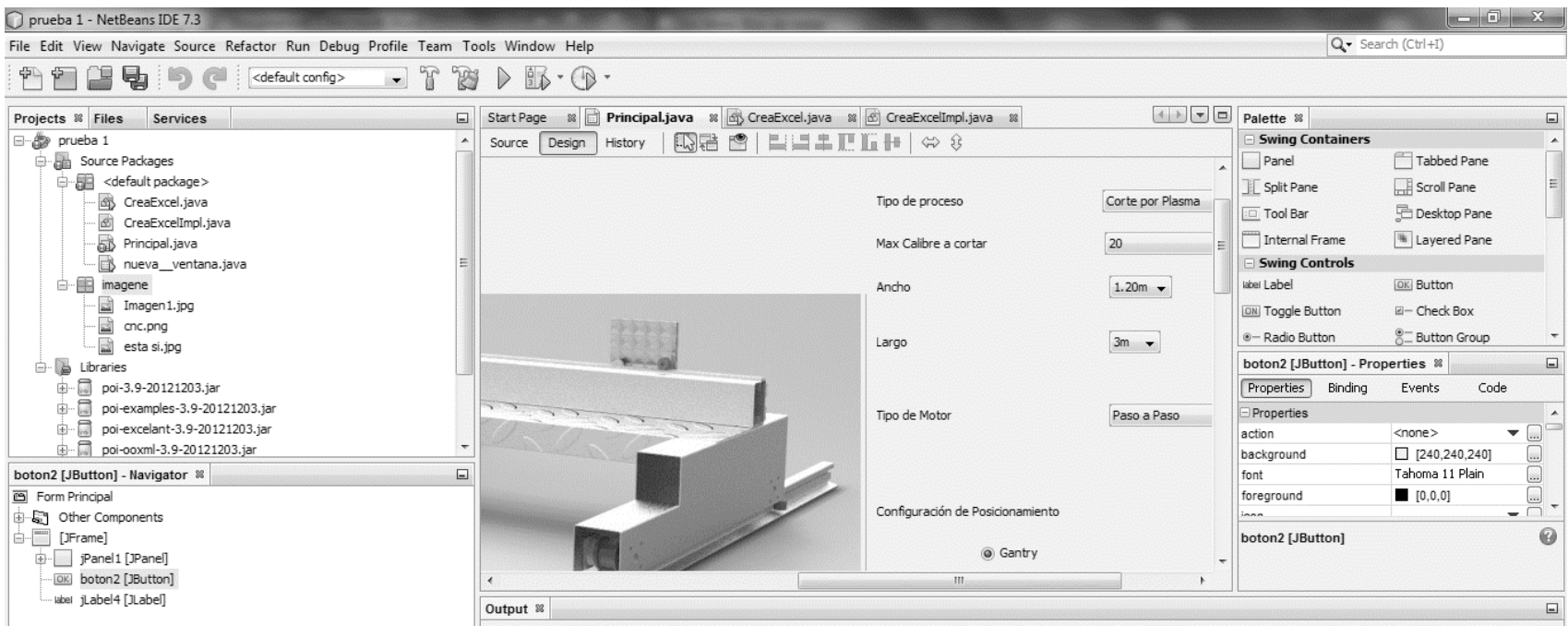

Figura 3. Diseño de la interfaz de usuario en el software NetBeans 7.3.

Como se observa, las dimensiones requeridas por Solid Edge para el diseño y actualización de la geometría del sistema corresponden al ancho de la lámina a cortar y el espesor de la misma, que junto con el método de corte, definen las variables relevantes para alimentar la interfaz de usuario; además, se disponen para seleccionar 4 tipos de procesos de manufactura: corte por oxiacetileno, corte por chorro de agua, corte por láser y corte por plasma; el calibre de la lámina a cortar se encuentra entre $10 \mathrm{~mm}$ y $20 \mathrm{~mm}$ para tres tamaños de ancho de lámina: 1200 mm, 1800 mm y 2400 $\mathrm{mm}$.

Dependiendo del calibre de la lámina se selecciona la herramienta que posea la potencia necesaria para realizar el trabajo, posteriormente se selecciona el tipo de motor, teniendo en cuenta la máxima velocidad de avance deseada y finalmente se selecciona el tipo de mecanismo para movilizar los ejes.

A continuación se describen cada uno de los subsistemas que conforman el equipo a diseñar mediante la herramienta informática y cómo a partir de los datos de entrada en la interfaz, se llega a obtener el diseño mecánico estructural y de posicionamiento, además de los componentes electrónicos y de control.
2.3 Diseño mecánico del sistema de posicionamiento

El desarrollo de este trabajo considera el uso de un sistema referencial cartesiano tridimensional, con movimiento en cada eje gobernado de forma independiente, teniendo en cuenta que el corte de material se hace sobre el plano horizontal $X$ e $Y, y$ que además, existe un desplazamiento vertical en dirección Z para prevenir que el actuador choque con la lámina y controlar la calidad del corte.

\section{Criterios de diseño mecánico}

El diseño propuesto tiene en cuenta las recomendaciones hechas en Chen, Chen, Lin y Changchien (1998) y en Amadori (2012), respecto a la aplicación de metodologías de diseño para ensamblaje y diseño para manufactura en el diseño concurrente de componentes mecánicos, diseño paramétrico de geometrías; además, en Chang, Wysk y Wang (1998), se describen algunos objetivos de diseño de un producto entre los cuales se encuentra el que sea fácil y económicamente manufacturado; para ello se establecen una serie de recomendaciones tales como: minimizar el número de partes, desarrollar un diseño modular, usar componentes estándar, establecer componentes multifuncionales, seleccionar materiales y procesos de manufac- 
tura óptimos, establecer una ruta de ensamble de las piezas y asumir tolerancias apropiadas.

A partir de estas recomendaciones se seleccionan materiales y procesos de manufactura, que brinden un bajo costo de producción y la certeza de que sea posible la implementación de las máquinas; con esto el sistema de posicionamiento diseñado responde al movimiento requerido por una máquina cortadora de lámina de metal CNC.

Como criterios de diseño se asume la implementación de un sistema robusto y estético. Por estas razones se elige lámina HR para la estructura y materiales estándar en la selección de los mecanismos de transmisión de movimiento. Así mismo, se asume una restricción para el área de trabajo de acuerdo a los tamaños disponibles comercialmente para láminas de acero a cortar, las cuales tienen 3 metros de largo por $1,20 \mathrm{~m}, 1,80 \mathrm{~m}$ y $2,40 \mathrm{~m}$ de ancho según sea el caso; generando así 3 tipos posibles de tamaño de las máquinas.

Los requerimientos comunes de cada sistema en lo referente a tamaño, peso, u otros parámetros que intervienen en la calidad de un producto son mostrados en la tabla 1:

\begin{tabular}{ll}
\hline \multicolumn{2}{c}{ Características de las máquinas cortadoras de lámina CNC } \\
\hline Área de trabajo & Máxima velocidad de trabajo \\
Área de la mesa & Tipo de Motores en los ejes \\
Precisión de posicionamiento & Dimensiones de embalado \\
Precisión de reposición & Peso neto \\
Resolución X-Y & Voltaje de operación \\
Perfil del Marco & Lenguaje de programación \\
Estructura de los ejes XY Z & Compatibilidad de software \\
Máximo consumo de energía & Máxima velocidad de avance \\
\hline
\end{tabular}

Tabla 1. Especificaciones técnicas comunes en un sistema cartesiano de posicionamiento.

El sistema de posicionamiento seleccionado para el diseño de equipos es de tipo pórtico, debido a los parámetros de diseño mecánico para la ubicación de un punto en un sistema cartesiano tridimensional. Este estilo de configuración mecánica, mueve la herramienta de la máquina de corte en un plano horizontal compuesto por un eje $X$ y un eje $Y$. En la figura 4 se puede observar un ejemplo de robot cartesiano tipo pórtico, en el cual se detalla cada una de sus partes: dos controladores lineales horizontales, un controlador lineal vertical, unos sensores de posición en el inicio y final de cada eje, un componente flexible para transportar cable al cabezal, unos reductores mecánicos que transmiten adecuadamente el movimiento disminuyendo el efecto inercial y finalmente unos servomotores en cada eje con sistemas de control a lazo cerrado para aumentar la confiabilidad y rendimiento del equipo (Overby, 2010).

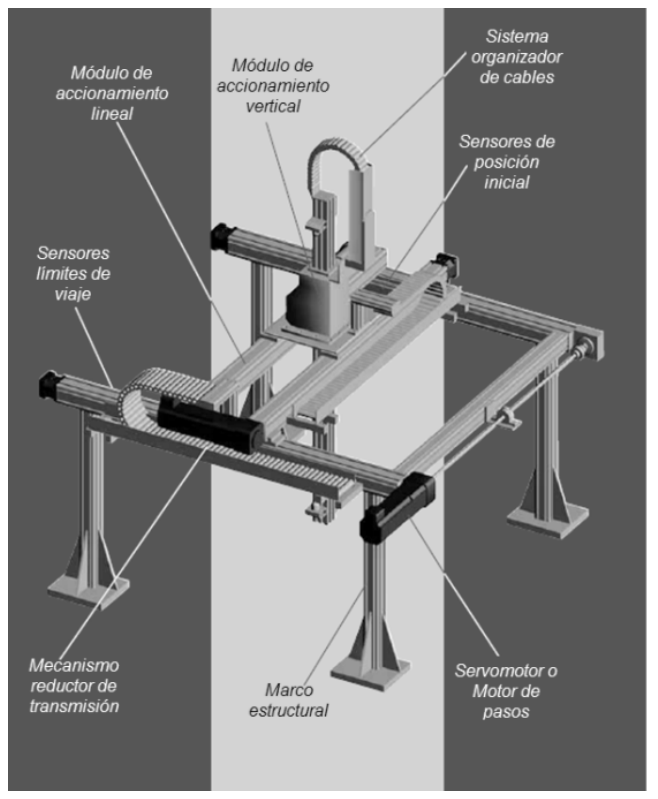

Figura 4. Sistema de posicionamiento tipo pórtico (Parker Hannifin Corporation, 2013). 


\section{Diseño paramétrico del sistema mecánico}

El sistema mecánico diseñado en Solid Edge, responde a una configuración parametrizada de cada pieza y conjunto, asociados de tal forma que un cambio de las cotas principales, refleja un cambio automático de la estructura completa (Wang, Meléndez, \& Tsai, 2008). Las dimensiones principales de la estructura están referenciadas a una spreadsheet de MS Excel, que contiene una base de datos de cada componente de la máquina (Tarkian, Ölvander, \& Lundén, 2008).
El diseño paramétrico del sistema mecánico se realizó en el software Solid Edge st6, aprovechando la característica de comunicación dinámica de datos entre el software MS EXCEL y la vinculación de valores de cotas a casillas en un archivo de EXCEL. En la figura 5 se muestra la sección de vínculos en el árbol de características del modelo, además de la tabla de variables que posee el ensamble general. En este caso se desea el menor número de variables a configurar para que el diseño se adapte a las especificaciones de ancho de la máquina impuestas en la interfaz de usuario.

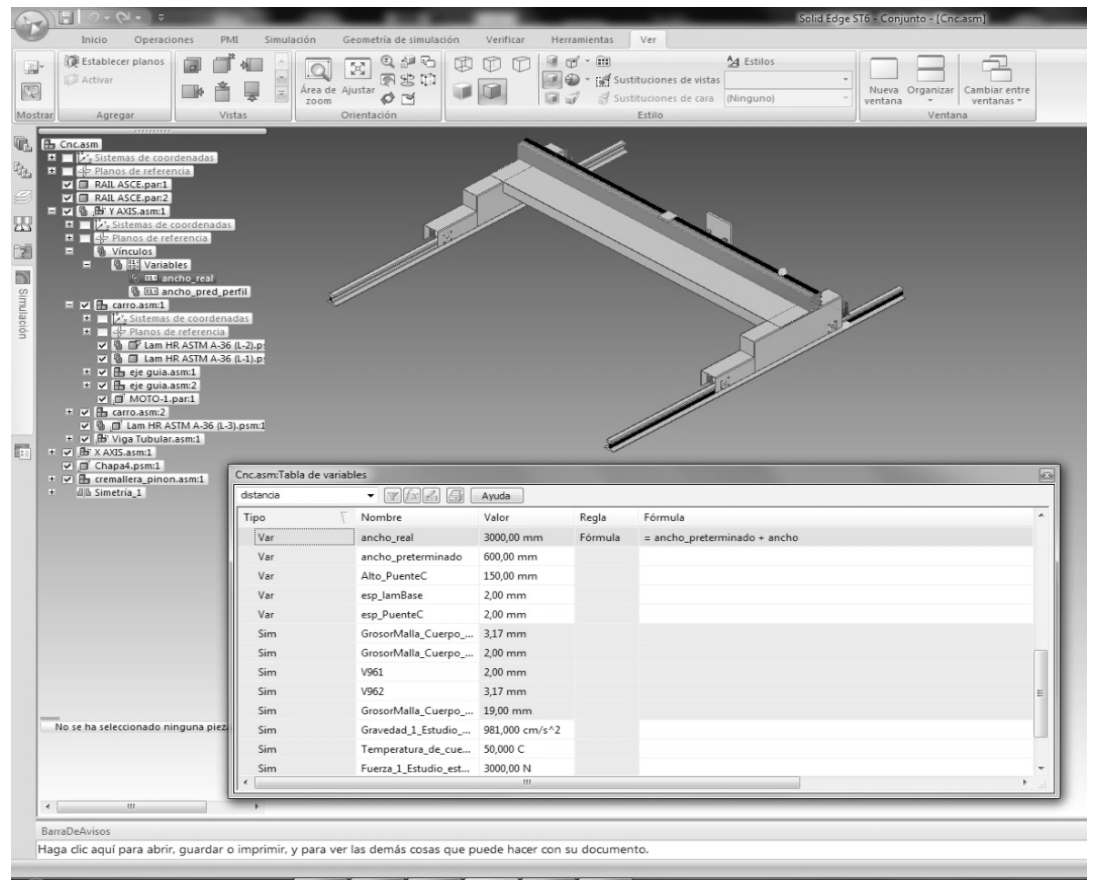

Figura 5. Variables del ensamble general del sistema mecánico.

\section{Mecanismos de trasmisión de movimiento}

I. Movimiento en el eje $X$ : Este movimiento está gobernado por un motor y un mecanismo piñón-cremallera, que mueve una masa determina- da por la suma de los componentes de la antorcha de corte, su estructura soporte y mecanismo de movimiento vertical; el movimiento está guiado por dos rieles lineales por los cuales se desplazan 4 rodillos por parejas para darle estabilidad al sistema, como se muestra en la figura 6 . 


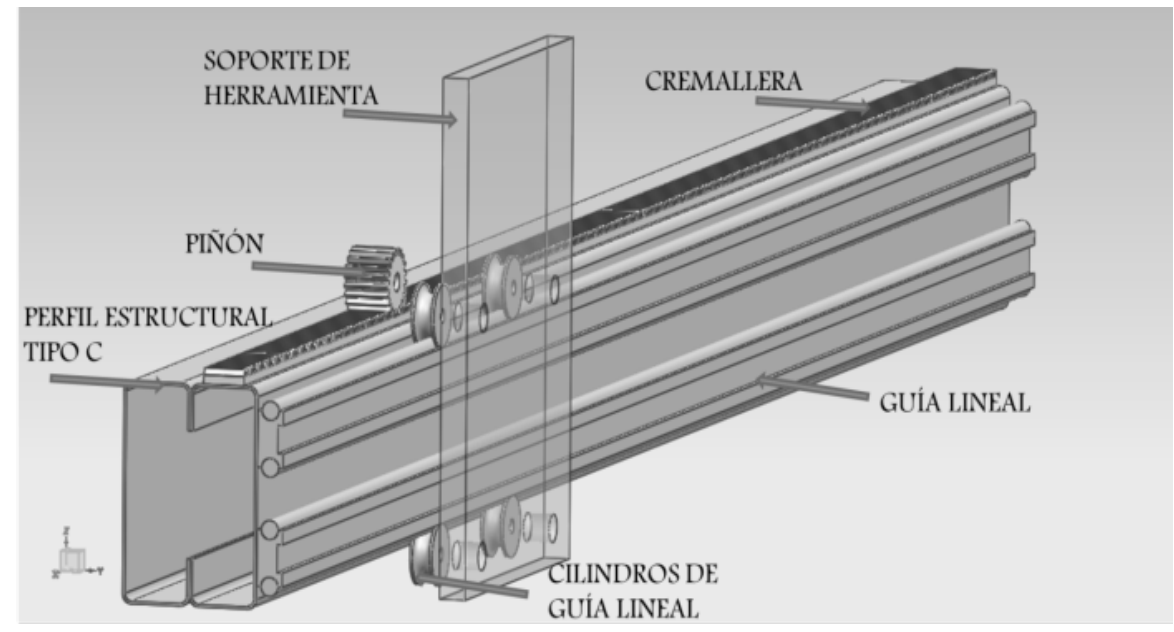

Figura 6. Actuador para movimiento en el eje $x$.

II. Movimiento en el eje $Y$ : Este movimiento está gobernado por dos motores y un sistema de transmisión de movimiento, integrado por dos mecanismos de piñón-cremallera al lado izquierdo y derecho del eje. Los motores proporcionan un movimiento rotacional que es transformado en lineal en el sentido de los rieles al interactuar los piñones con las cremalleras que transmiten el mo- vimiento en la dirección $\mathrm{Y}$ al eje $\mathrm{X}$ acoplado a este. Sobre los rieles se disponen 4 rodamientos por parejas sobre cada uno, esto para permitir la rotación y disminuir la fricción existente. Uniendo los dos brazos se encuentra una lámina que soporta el eje $X$, el equipo de corte y la estación de energía para la máquina. La configuración del eje $Y$ se muestra en la figura 7.

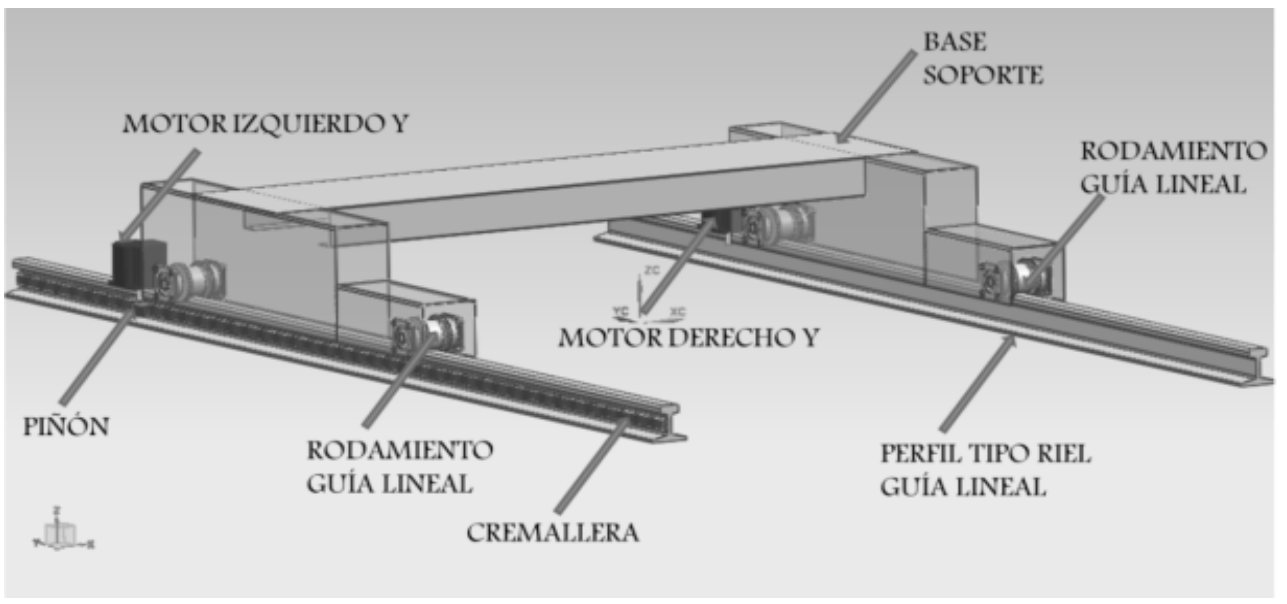

Figura 7. Actuador para movimiento en el eje Y.

La figura 8 muestra un diseño renderizado del ensamble final del modelo elaborado en el software Solid Edge ST6. La estructura soporta los 2 ejes de transmisión de movimiento, la base para la herra- mienta de corte se diseña de acuerdo a criterios de portabilidad, volumen, anclaje, y rigidez final; permitiendo el movimiento libre de los ejes. Se observa como sobre el eje $Y$ se encuentra monta- 
do el eje $X, y$ que estos dos ejes en movimiento simultáneo sobre un plano realizan la geometría del corte, además el eje $\mathrm{X}$ tiene la herramienta final ensamblada sobre él.

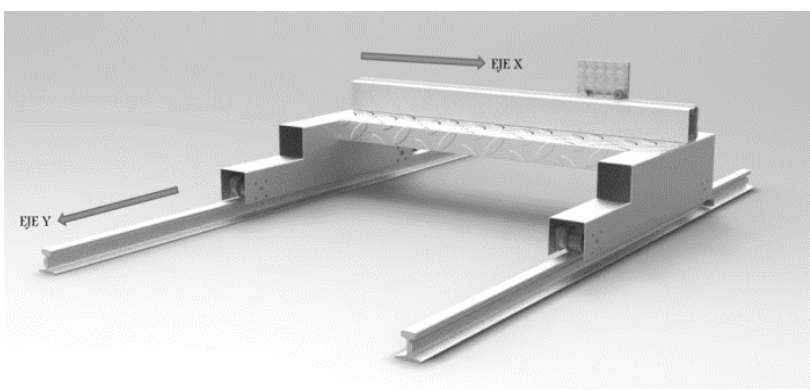

Figura 8. Prototipo virtual de la configuración mecánica de la máquina en software CAD.

\subsection{Selección del sistema de control electrónico}

El Sistema electrónico para el control describe los componentes de actuación y sensado utilizados en la máquina CNC. A partir de los diferentes tipos de control e interfaces de posicionamiento cartesiano coordenado, se determinó la estrategia más eficiente y se identificó la respuesta adecuada de los actuadores dentro del mecanismo.

El sistema de control que gobierna el sistema mecánico está basado en un sistema informático que, a través de un software maestro, envía información a una tarjeta de control, la cual se encarga de interpretarla y emitir señales de salida a los circuitos de amplificación de potencia para el control de los actuadores; finalmente unos sensores realizan una realimentación a la tarjeta controladora para verificar el cumplimiento del algoritmo de control (Barahona, León-Medina, \& Díaz, 2013).

El sistema se compone de los siguientes elementos básicos: controlador, amplificador, actuador y sensores. En esta investigación se adoptó la tecnología de control de movimiento programable, PMC por sus siglas en inglés, la cual define la aplicación de software y hardware programable en conjunto con dispositivos de entrada sensoriales, actuadores y otros dispositivos de realimentación para el control de uno o más movimientos lineales o rotativos.

El controlador incluye un medio para ingresar un conjunto de instrucciones en su memoria, que luego se traducen en una serie de impulsos eléctricos y una salida a un amplificador para el control de algún tipo de actuador. El amplificador recibe las señales desde el controlador y las aumenta a niveles apropiados para el funcionamiento del actuador.

Los actuadores permiten el movimiento físico real y están estrechamente acoplados a las características de diseño del amplificador. El conjunto amplificador/actuador puede ser cualquiera de varias clasificaciones de diseño diferentes, pero se recomienda al diseñador utilizar hardware y software de marca compatible para asegurar la correcta comunicación entre dispositivos. Aprovechando las ventajas del uso de un sistema de control maestro esclavo, donde un computador se comunica con una tarjeta controladora externa para vincular y coordinar la función de control de movimiento, se plantea el sistema de control de movimiento que aparece en la figura 9.

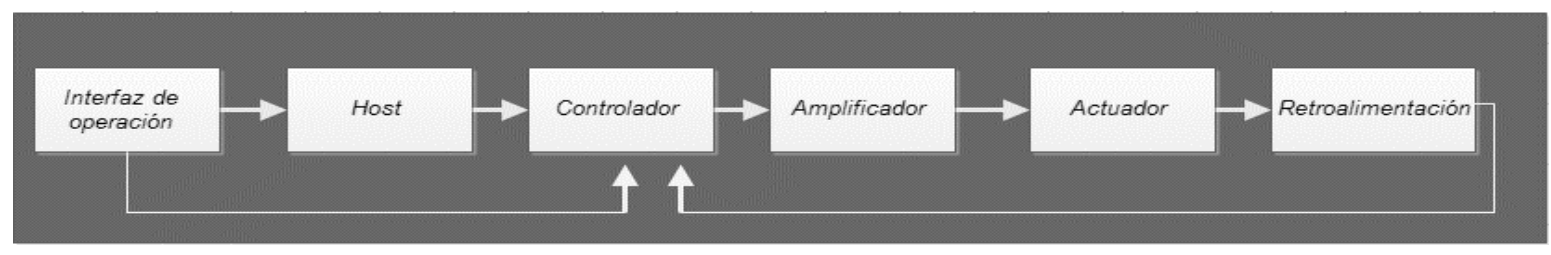

Figura 9. Diagrama de bloques del sistema de control para el sistema cartesiano. 


\section{Tarjeta controladora principal}

La tarjeta controladora es programada para la interpretación del código de máquina que seguirá las trayectorias dadas desde un computador, el manejo de la comunicación entre el computador, la recepción de las señales de los sensores y la emisión de las señales para la actuación. La tarjeta trabaja como cerebro del sistema manejándolo a través de las entradas y salidas que pueden ser analógicas o digitales. Dicha tarjeta debe satisfacer algunos requerimientos como: una velocidad de procesamiento adecuada para el trabajo, buena confiabilidad en la comunicación y contar con un número suficiente de entradas y salidas para enlazar los actuadores y sensores.

\section{Circuitos controladores de potencia para los moto-} res

Las señales generadas por la tarjeta de control son enviadas a tarjetas electrónicas que las codifican y manejan la potencia de cada uno de los motores de la máquina. La tarjeta controladora de cada motor permite controlar la corriente existente en los motores, trabajar con una señal de medio paso para aumentar la precisión de los motores o inclusive trabajar micro paso, esto para motores paso a paso, y recibir el flujo de información enviado por la tarjeta de control, consistente en tres señales que definen la dirección, el paso y la activación de cada motor. Para servomotores, el controlador de potencia generalmente se encarga de recibir la señal de un encoder y realizar un control PID sobre la salida de paso hacia el motor.

Software de control para sistemas cartesianos automatizados

Es importante entender que hay diferentes tipos de software que se utilizan en el proceso de corte de una lámina. Hasta tres programas diferentes se pueden utilizar para producir una sola parte (Tec- no Inc., 2013). A continuación se analiza la cadena de flujo de información desde el diseño inicial de la pieza hasta la comunicación con la planta y los actuadores del sistema cartesiano para obtener al final la pieza fabricada. La cadena de información es mostrada en la figura 10.

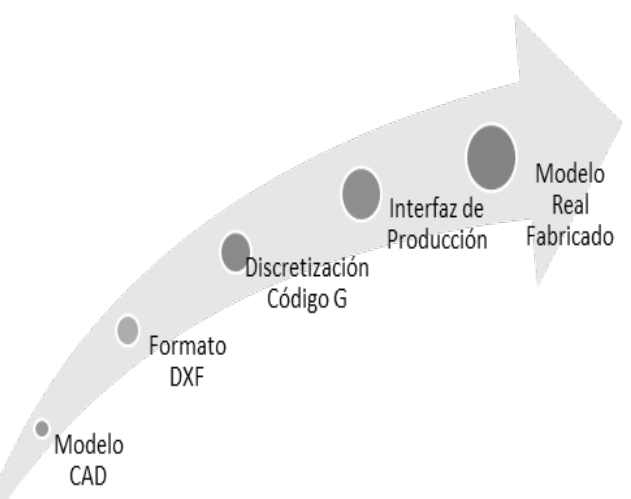

Figura 10. Cadena de flujo de información en el proceso de corte.

Inicialmente se utiliza un programa CAD para crear la geometría de la pieza a cortar, p. ej. AutoCAD o cualquier otro programa de dibujo asistido por computador. Posteriormente la geometría de la pieza se importa en un programa CAM, para generar las trayectorias a cortar; en él se puede emular el mecanizado propuesto y generar el código de máquina final que será cargado finalmente por el software HOST que comanda el equipo. Este último programa se utiliza para conducir la antorcha manualmente, establecer el origen de la herramienta, ejecutar el código de máquina y finalmente realizar el corte de la pieza.

\section{Resultados y discusión}

Para probar la herramienta de diseño de sistemas cartesianos de posicionamiento, se eligieron los parámetros iniciales mostrados en la tabla 2: 


\begin{tabular}{ll}
\hline Parámetro de entrada & Selección \\
\hline Tipo de Proceso & Corte por plasma \\
Máximo calibre de lámina a cortar & 20 \\
Ancho & $1.20 \mathrm{~m}$ \\
Largo & $3 \mathrm{~m}$ \\
Tipo de motor & Paso a paso \\
Configuración de posicionamiento & Gantry \\
Mecanismo de transmisión & Cremallera piñón \\
Máxima velocidad de avance & $50 \mathrm{~cm} / \mathrm{s}$ \\
\hline
\end{tabular}

Tabla 2. Parámetros de entrada para el diseño de equipo ejemplo.

En la figura 11 se ilustra una primera impresión del modelo cartesiano diseñado, el eje $Y$ apoyado sobre dos rieles y el eje $\mathrm{X}$ apoyado sobre el eje $\mathrm{Y}$.

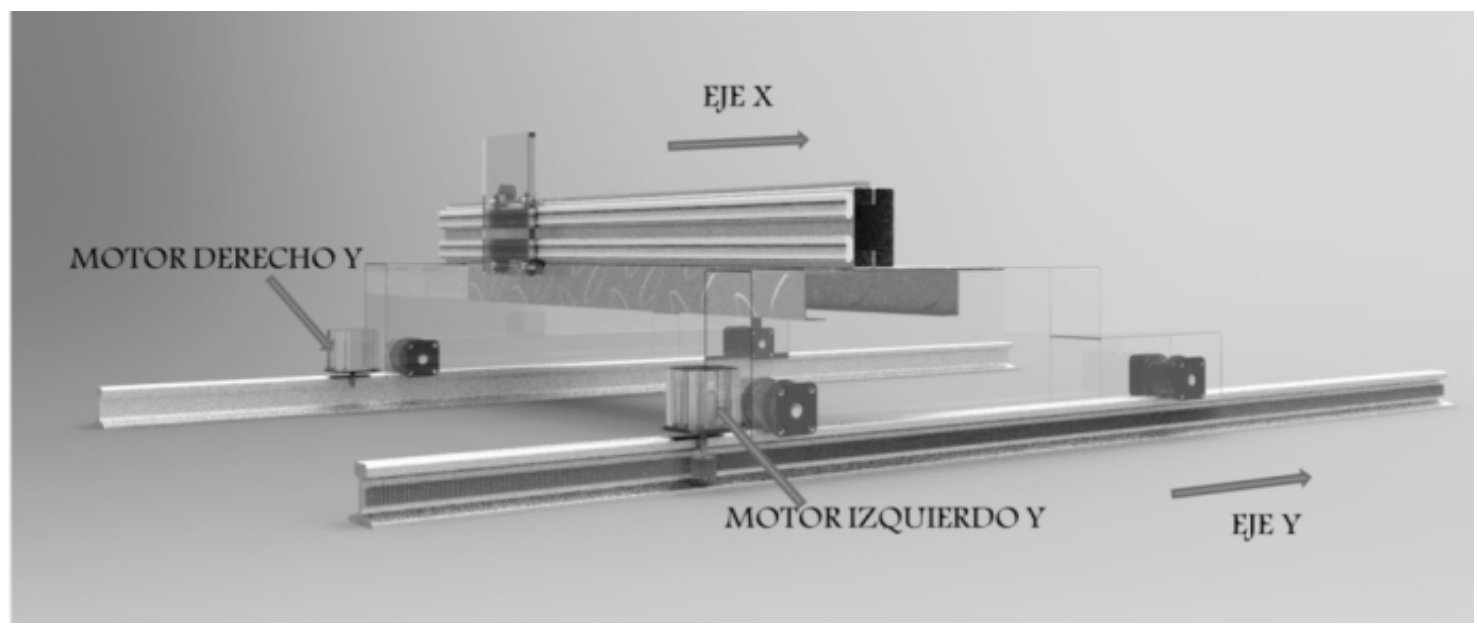

Figura 11. Disposición de los ejes $X$ e $Y$ en el modelo final diseñado.

El modelo fue ensamblado en Solid Edge y referenciado de tal forma que dada la entrada de un valor de ancho este se actualice automáticamente. Lo anterior se consigue al establecer una relación fija de distancia entre los planos de las piezas del ensamble. La lámina base que soporta el equipo de corte tiene una distancia fija a los carros del eje Y igual a $1425 \mathrm{~mm}$, la cual resulta del cálculo dado en la variable V417 mostrada en la figura 12. 


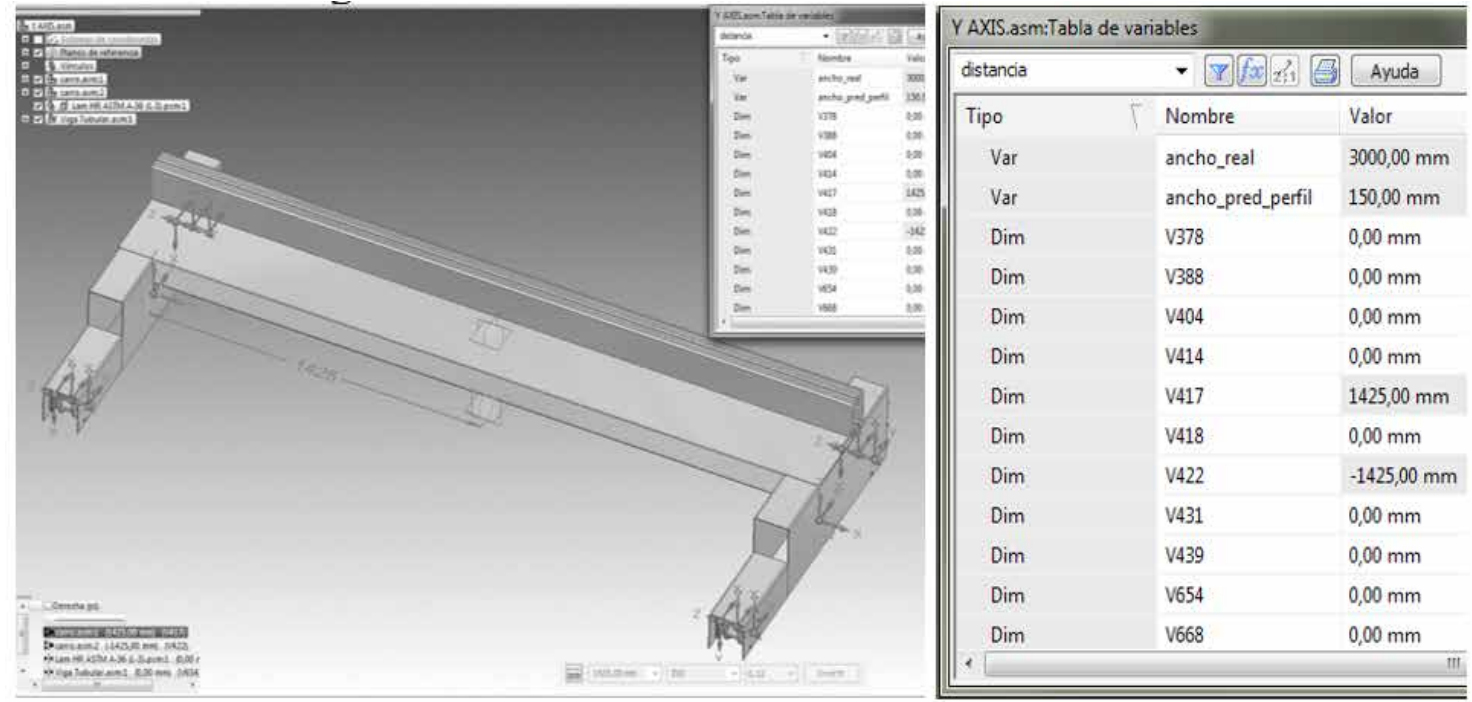

Figura 12. Eje y parametrización de la distancia de uno de los componentes del sistema diseñado.

La interfaz de usuario final de la herramienta de diseño se ilustra en la figura 13.

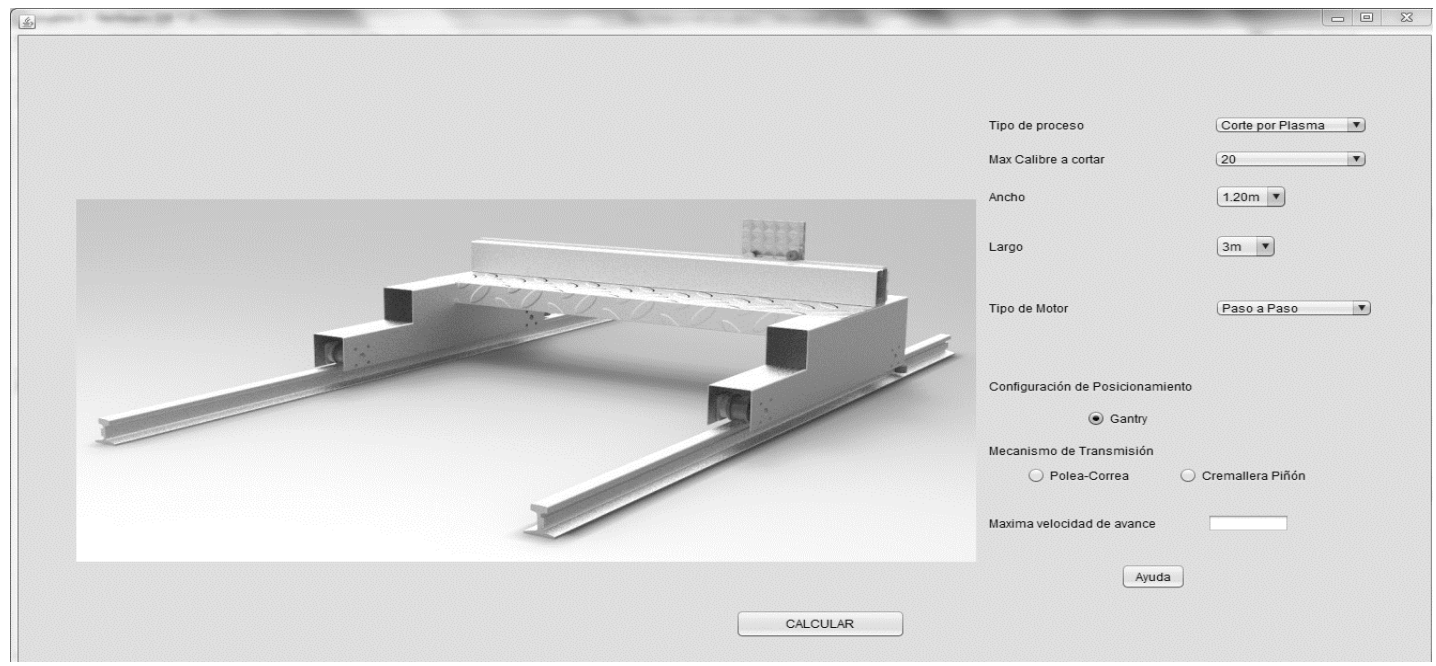

Figura 13. Diseño final de la Interfaz de usuario de la herramienta de diseño.

Finalmente, las características finales de la máquina de corte por plasma son encontradas por la herramienta de diseño; una muestra de dichas características se evidencia en la tabla 3.

\begin{tabular}{ll}
\hline Parámetro de salida & Selección \\
\hline Software Generador de código de máquina & Sheetcam \\
Software Maestro & Mach3 \\
Motor X & Nema 42 \\
Motor Y & Nema 42
\end{tabular}


Motor Z

Control de altura de antorcha (THC)

Tarjeta controladora principal CNC

Tarjeta controladora de motores

Herramienta de corte por plasma
Nema 34

Texas MicroCircuits - THC301 digital

PhantomTM II Shape Cutting Controller

GM215 Step Motor / Motion Controller Gecko-

drive

HySpeed Plasma HSD130

Tabla 3. Especificaciones finales entregadas por la herramienta de diseño de un equipo ejemplo.

\section{Conclusiones}

El uso de la herramienta de diseño aquí reportada, permite realizar los cálculos necesarios para seleccionar los componentes mecánicos, electrónicos y de control, pertenecientes a un sistema de posicionamiento; además se puede seleccionar la herramienta de trabajo para cada sistema, teniendo en cuenta las características particulares de cada proceso.

La integración de diversas herramientas informáticas en el diseño de los diferentes componentes de máquinas de fabricación digital, hace posible el flujo de información desde la entrada de los requerimientos iniciales hasta la entrega final de las especificaciones técnicas para la construcción de un equipo diseñado.

A partir del análisis de la información referente a la fabricación digital se consolida una base del conocimiento, que amplía los recursos de automatización y control de procesos de manufactura trabajados al interior del grupo de investigación, en lo referente a sistemas de control de movimiento y control numérico computarizado.

\section{Agradecimientos}

Este trabajo fue apoyado por el Departamento Administrativo de Ciencia, Tecnología e Innovación, Colciencias, y el Programa Jóvenes Investigadores e Innovadores 2012.

\section{Referencias}

Amadori, K. (2012). Geometry based design automation applied to aircraft modelling and optimization (Tesis doctoral). Linköping University, Suecia.

Apache Software Foundation. (2013). Apache POI the Java API for Microsoft documents. Recuperado de http://poi.apache.org/

Barahona, E. A. T., León-Medina, J. X., \& Díaz, E. T. (2013). Sistema de posicionamiento aplicado a la técnica de impresión $3 \mathrm{~d}$ modelado por deposición fundida. Revista de investigación, desarrollo e innovación, 3(1), 25-32. doi: 10.19053/20278306.2135

Chang, T., Wysk, A., \& Wang, H. (1998). Computer-aided manufacturing. (2da ed.).New Jersey, Estados Unidos: Prentice Hall.

Chen, K. H., Chen, S. J. G., Lin, L., \& Changchien, S. (1998). An integrated graphical user interface (GUI) for concurrent engineering design of mechanical parts. Computer integrated manufacturing systems, 11(1), 91-112. doi: 10.1016/S0951-5240(98)000160

Chryssolouris, G., Mavrikios, D., Papakostas, N., Mourtzis, D., Michalos, G., \& Georgoulias, K. (2009). Digital manufacturing: history, perspectives, and outlook. Proceedings of the Institution of Mechanical Engineers, Part B: Journal of Engineering Manufacture, 223(5), 451-462. Recuperado de: http:// pib.sagepub.com/content/223/5/451.abstract 
International Organization for Standardization. (2002) Thermal cutting -- classification of thermal cuts -- geometrical product specification and quality tolerances (ISO 9013:2002). Recuperado de: http:// www.iso.org/iso/catalogue_detail.htm?csnumber $=29998$

León-Medina, J. X. (2014). Herramienta de diseño para sistemas cartesianos de posicionamiento tridimensional aplicable en equipos de fabricación digital del sector automotriz. Informe final de proyecto convocatoria joven investigador Colciencias 2012, Universidad Pedagógica y Tecnológica de Colombia, seccional Duitama, Colombia.

Overby, A. (2010).CNC machining handbook: building, programming, and implementation. McGraw-Hill, Inc.

Parker Hannifin Corporation. (2013). Electromechanical automation division, gantry systems. Recuperado de: http://www.parkermotion.com/literature/precision_cd/CD-EM/daedal/cat/english/ Gantry\%20Systems.pdf

Sarmiento, M.Y., \& Rojas, J. (2006). Construcción de una máquina para corte térmico de siluetas metálicas asistida por computador. Revista Colombiana de Tecnologías de Avanzada, 1(7), 44-47. Recuperado de: http://www.unipamplona.edu.co/unipam-
plona/portallG/home_40/recursos/02_v07_12/ revista_07/16112011/v07_09.pdf

Tarkian M., Ölvander J., \& Lundén B. (2008). Integration of parametric CAD and dynamic models for industrial robot design and optimization. Proceedings of the ASME 2008 International Design Engineering Technical Conferences \& Computers and Information in Engineering Conference IDETC/CIE , 3, 761-769. doi: 10.1115/DETC2008-49919

Tecno Inc. (2013). Tecno CNC plasma cutting. Recuperado de http://www.techno-isel.com/education1/Plasma_Cutter.htm

Wang S., Meléndez S., \& Tsai C. (2008). Application of parametric sketching and associability in 3D CAD. Computer-Aided Design \& Applications, 5(6), 822-830. doi: 10.3722/cadaps.2008.822-830

Westkämper, E. (2007). Digital Manufacturing in the global era. En Cuhna, D.F. \& Maropoulus, P.G. (Eds.), Digital Enterprise Technology (pp. 3-14). Nueva York, Estados Unidos: Springer.

Wilfert, H. G., \& Seeland, H. (1986). CAD/CAM: integration in the automobile industry. En Encarnação, J., Schuster, R., \& Vöge, E. (Eds.), Product Data Interfaces in CAD/CAM Applications (pp. 34-62). Munich, Alemania: Springer. 